\title{
Effects of SGLT2 Inhibition on eGFR and Glomerular and Tubular Damage Markers in Japanese Patients With Type 2 Diabetes
}

\author{
Hiromi Konishi ${ }^{a}$, Jun Shirakawa ${ }^{\text {a, b }}$, Kazuki Tajima ${ }^{a}$, Tomoko Okuyama ${ }^{\text {a }}$, Yu Togashia ${ }^{\text {, }}$ \\ Hikaru Takamine ${ }^{\mathrm{a}}$, Yoshinobu Kondo ${ }^{\mathrm{a}}$, Rieko Kunishita ${ }^{\mathrm{a}}$, Yasuo Terauchi ${ }^{\mathrm{a}}$, b
}

\begin{abstract}
Background: Sodium-glucose cotransporter 2 (SGLT2) inhibitors are a new class of antihyperglycemic drugs that enhances insulin-independent urinary glucose excretion. Recent studies have suggested that SGLT2 inhibitors possess a renoprotective property in type 2 diabetes patients. However, evidence of the effects of SGLT2 inhibition on glomerular and tubular damage markers is lacking. The aim of this study was to examine the effect of SGLT2 inhibitors on renal function, especially on glomerular and tubular damage markers in patients with type 2 diabetes.
\end{abstract}

Methods: We retrospectively analyzed data from 81 patients who used SGLT2 inhibitors. Next, we investigated whether treatment with SGLT2 inhibitors affected urinary damage markers including $\mathrm{N}$-acetyl- $\beta$-D-glucosaminidase (NAG), liver-type fatty acid-binding protein (L-FABP), type IV collagen, and $\beta 2$-microglobulin ( $\beta 2 \mathrm{MG})$ in patients with type 2 diabetes.

Results: In the retrospective study, SGLT2 inhibition reduced the estimated glomerular filtration rate (eGFR) at 4 and 12 weeks in a manner that was correlated with the baseline eGFR. In the longitudinal study, SGLT2 inhibition tended to increase the urinary damage marker levels with an accompanying decrease in eGFR after 1 month of use. The observed changes in eGFR and urinary damage markers were reversed at 3 months, even though both the HbAlc level and blood pressure were further improved.

Conclusions: These results indicated that SGLT2 inhibition reduces the eGFR in a manner depending on the baseline eGFR levels and transiently increased the glomerular and tubular damage markers in

Manuscript submitted August 6, 2018, accepted September 10, 2018

aDepartment of Endocrinology and Metabolism, Graduate School of Medicine, Yokohama City University, Yokohama, Japan

${ }^{\mathrm{b} C}$ Corresponding Author: Jun Shirakawa, Department of Endocrinology and Metabolism, Graduate School of Medicine, Yokohama City University, 3-9 Fukuura, Kanazawa-ku, Yokohama 236-0004, Japan. Email: jshira-tky@ umin.ac.jp; Yasuo Terauchi, Department of Endocrinology and Metabolism, Graduate School of Medicine, Yokohama City University, 3-9 Fukuura, Kanazawa-ku, Yokohama 236-0004, Japan. Email: terauchi-tky@umin.ac.jp

doi: https://doi.org/10.14740/jem531w patients with type 2 diabetes.

Keywords: Glomerular and tubular damage markers; SGLT2 inhibitor; Type 2 diabetes

\section{Introduction}

Sodium-glucose cotransporter 2 (SGLT2) inhibitors are a new class of antihyperglycemic drug that enhances insulin-independent urinary glucose excretion without increasing body weight. Recent evidence suggests that SGLT2 inhibitors reduce the risk of cardiovascular events and exert renoprotective effects [1]. Studies have suggested that the renoprotective actions of SGLT2 -inhibitors are caused by reductions in blood glucose, body weight, and blood pressure levels [2-4], with tubuloglomerular feedback (TGF) playing a particularly important role in the renal effects [5, 6]. Hyperglycemia can enhance glomerular filtration rate (GFR) during the early stage of diabetes, and individuals with glomerular hyperfiltration are thought to be at risk for progression to diabetic nephropathy [7]. Diabetes-induced proximal tubule growth involves an initial hyperplastic growth period and a hypertrophic growth period [8]. Another study also suggested that tubular growth is related to an enhanced proximal tubular fluid reabsorption rate [9]. Thus, in diabetic nephropathy, proximal tubule function is enhanced by tubular growth, and TGF is thought to induce glomerular hyperfiltration. Recent animal studies have reported that an SGLT2 inhibitor improved glomerular hyperfiltration independent of a reduction in blood glucose levels [10], but that tubular growth was inhibited by glycemic control [6]. Treatment with dapagliflozin resulted in an initial fall in the estimated glomerular filtration rate (eGFR), but this reduction was completely reversible only 1 week after dapagliflozin discontinuation in type 2 diabetes patients [11]. The SGLT2 inhibitor empagliflozin reportedly reduced the GFR in type 1 diabetes patients with hyperfiltration of the normal GFR [5]. The EMPA-REG OUTCOME trial also showed a reduction in eGFR after 4 weeks in patients with type 2 diabetes [1], while canagliflozin decreased the eGFR after 3 weeks of treatment in type 2 diabetes patients with stage 3 chronic kidney disease [12]. A subgroup of Asian pa- 
Table 1. Mean Changes From Baseline in Glycemic Control, Lipid Profile, and Renal Function in Patients Receiving SGLT2 Inhibitors for 12 Weeks $(\mathrm{N}=81)$

\begin{tabular}{|c|c|c|c|c|c|c|}
\hline & \multirow{2}{*}{ Baseline } & \multirow{2}{*}{4 weeks } & \multirow{2}{*}{12 weeks } & \multicolumn{3}{|c|}{ P value } \\
\hline & & & & 0 vs. 4 weeks & 4 vs. 12 weeks & 0 vs. 12 weeks \\
\hline Body weight (kg) & $78.3(68.0,90.6)$ & $77.4(66.7,90.0)$ & $76.2(67.0,89.0)$ & $<0.0001 *$ & $<0.0001^{*}$ & $<0.0001 *$ \\
\hline $\mathrm{HbAlc}(\mathrm{mmol} / \mathrm{mol})$ & $65(57,75)$ & $62(55,70)$ & $58(53,67)$ & $<0.0001 *$ & $<0.0001 *$ & $<0.0001 *$ \\
\hline HbA1c (\%) & $8.1(7.4,9.0)$ & $7.8(7.2,8.6)$ & $7.5(7.0,8.3)$ & $<0.0001^{*}$ & $<0.0001 *$ & $<0.0001 *$ \\
\hline $\mathrm{eGFR}\left(\mathrm{mL} / \mathrm{min} / 1.73 \mathrm{~m}^{2}\right)$ & $80.0(69.4,93.0)$ & $74.7(63.7,91.6)$ & $77.3(65.4,91.1)$ & $<0.0001^{*}$ & 0.37 & $<0.0001 *$ \\
\hline $\mathrm{CCr}(\mathrm{mL} / \mathrm{min})$ & $131(99.3,178)$ & $120(87.0,160)$ & $120(88.0,160)$ & $<0.0001^{*}$ & 0.44 & $<0.0001 *$ \\
\hline LDL-C (mg/dL) & $101(80.8,130)$ & $98.5(80.9,115)$ & $104(83.8,127)$ & $0.003^{*}$ & $0.0029 *$ & 0.49 \\
\hline HDL-C (mg/dL) & $50.0(41.8,58.3)$ & $48.5(42.0,57.5)$ & $52.0(43.0,59.5)$ & 0.28 & $0.0012 *$ & 0.096 \\
\hline
\end{tabular}

Mean \pm standard deviation or medians (25th and 75th percentiles). CCr was calculated by Cockcroft and Gault equation. P values were determined by paired $t$-test or Wilcoxon signed-rank test. * $\mathrm{P}<0.05$.

tients in the EMPA-REG OUTCOME trial showed reductions in the risk of cardiovascular mortality after treatment with SGLT-inhibitor, and an initial decrease in eGFR was also observed in Asian patients [13]. However, the clinical meaning of the initial reduction in eGFR induced by SGLT2 inhibition remains unclear in terms of glomerular and tubular functional impairment. In the present study, we first evaluated the effects of SGLT2 inhibitor on the changes in eGFR in Japanese type 2 diabetes patients. We also analyzed several glomerular and tubular damage markers, such as urinary N-acetyl- $\beta$-Dglucosaminidase (NAG), liver-type fatty acid binding protein (L-FABP), type IV collagen, and $\beta 2$ microglobulin ( $\beta 2 \mathrm{MG})$, in a subgroup of patients who were treated with an SGLT-2 inhibitor.

\section{Materials and Methods}

\section{Ethics approval and consent to participate}

The study was approved by the institutional review board and was registered with the University Hospital Medical Information Network Japan (UMIN) (ID\# 000021619). The patients were anonymized to protect their personal information. The study protocol was approved by the Medical Ethics Committee of the Yokohama City University Hospital (Reference No. B151001013 and B151105005), and Yokohama Central Hospital (on July 14, 2016), and the study was performed in accordance with the Declaration of Helsinki. All methods were carried out in accordance with relevant guidelines and regulations. All subjects of prospective study enrolled gave written informed consent for participation. As a retrospective study, the opt-out method of obtaining informed consent was adopted.

\section{Study population}

We screened all the patients with a history of SGLT2 inhibitor use at Yokohama City University Hospital. This retrospective study analyzed the medical records data of patients with type 2 diabetes who received SGLT2 inhibitors at Yokohama City University Hospital between April 2014 and November 2016. During these 2 years and 7 months, a total of 166 patients received an SGLT 2 inhibitor at least once (ipragliflozin: 56 patients, dapagliflozin: 32 patients, tofogliflozin: 13 patients, luseogliflozin: 13 patients, canagliflozin: 30 patients, empagliflozin: 22 patients). We analyzed changes in body weight, HbA1c, eGFR, low-density lipoprotein (LDL)-cholesterol, high-density lipoprotein (HDL)-cholesterol, triglyceride and uric acid.

Patients who visited at 4 and 12 weeks after the start of SGLT2 inhibitor treatment, and who underwent blood and urine tests were included in the present study. Patients who did not visit at the predefined intervals and who exhibited poor medication adherence were excluded. Finally, we analyzed data from 81 patients who met the inclusion/exclusion criteria. Based on the results of this retrospective study, we then investigated whether treatment with an SGLT2 inhibitor affected the eGFR and urinary damage markers including NAG, L-FABP, type IV collagen, and $\beta 2 \mathrm{MG}$ in patients with type 2 diabetes.

Japanese type 2 diabetes patients who had not been previously treated with an SGLT2 inhibitor were recruited from among inpatients or outpatients of Yokohama City University Hospital or Yokohama Central Hospital. Patients with a low eGFR $\left(<29 \mathrm{~mL} / \mathrm{min} / 1.73 \mathrm{~m}^{2}\right)$ or kidney diseases other than diabetic nephropathy were excluded. Twenty-three eligible patients (10 patients for a prospective study and 13 patients for a retrospective observational study) were the subjects of the final analysis. The patients received tofogliflozin $(20 \mathrm{mg} /$ day $)$, 
and the eGFR, NAG, L-FABP, type IV collagen, and $\beta 2 \mathrm{MG}$ levels were assessed at baseline and at the end of the forth and 12 th weeks. The 23 patients were followed up for 4 weeks, and 19 patients $(83 \%)$ were again followed up at 12 weeks.

\section{Measurements}

The HbA1c levels were measured using high-performance liquid chromatography (HPLC) (National Glycohemoglobin Standardization Program (NGSP) values). The urinary concentrations of microalbumin were measured using a turbidimetric immunoassay kit. The $\beta 2 \mathrm{MG}, \mathrm{NAG}$, L-FABP, and type IV collagen levels were determined using a latex-enhanced turbidimetric immunoassay kit (Denka Seiken Co., Tokyo, Japan), a 6-methyl-2-pyridyl-N-acetyl-1-thio- $\beta$-Dglucosaminide (MPT-NAG) kit (Nittobo Medical CO., Tokyo, Japan), a two-step sandwich enzyme-linked immunosorbent assay kit (CMIC Holdings Co., Tokyo, Japan), and a one-step sandwich enzyme-linked immunosorbent assay kit (Kyowa Pharma Chemical Co., Toyama, Japan), respectively. The estimated glomerular filtration rate was calculated as previously described [14]: eGFR $\left(\mathrm{mL} / \mathrm{min} / 1.73 \mathrm{~m}^{2}\right)=194 \times$ serum creatinine $(-1.094) \times$ age $(-0.287) \times 0.739$ (if female) .

\section{Statistical analysis}

Continuous data are presented as the mean \pm standard deviation (SD) or the median and interquartile range. For paired analyses, paired $t$-test for continuous values with a normal distribution was used. A Wilcoxon signed-rank test was used for continuous values that did not follow a normal distribution. For unpaired analysis, we use Mann-Whitney $U$ test for continuous values that did not follow a normal distribution. All the statistical analyses were performed using the Statistical Package for JMP 12.0.1 (SAS Institute Inc., Cary, NC, USA). A two-sided $\mathrm{P}$ value $<0.05$ was considered statistically significant.

\section{Results}

\section{SGLT2 inhibitor transiently attenuated eGFR in patients with type 2 diabetes when examined retrospectively}

Table 1 shows the parameters before and after 4 or 12 weeks of treatment with SGLT2 inhibitors in 81 type 2 diabetes patients (44 men and 37 women; mean age, $54.5 \pm 12.3$ years old; ipragliflozin: 22 patients, dapagliflozin: 14 patients, tofogliflozin: seven patients, luseogliflozin: 12 patients, canagliflozin: 21 patients, empagliflozin: five patients). SGLT2 inhibition reduced body weight, improved HbAlc, and decreased LDL cholesterol and uric acid levels significantly at 4 and 12 weeks (Table 1). The eGFR levels were significantly reduced after treatment with the SGLT2 inhibitor at week $4(-6.6 \%, \mathrm{P}<0.0001)$, while the levels of eGFR at week 12 tended to have recovered to the baseline level, compared with the level at week $4(-3.4 \%$ compared with baseline, $\mathrm{P}=0.37)$. Univariate and multivariate

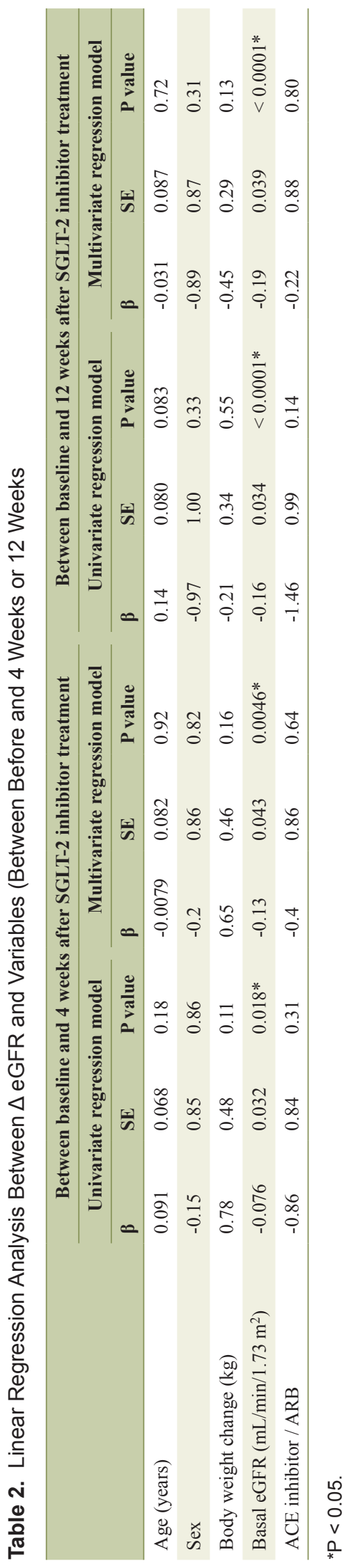


a

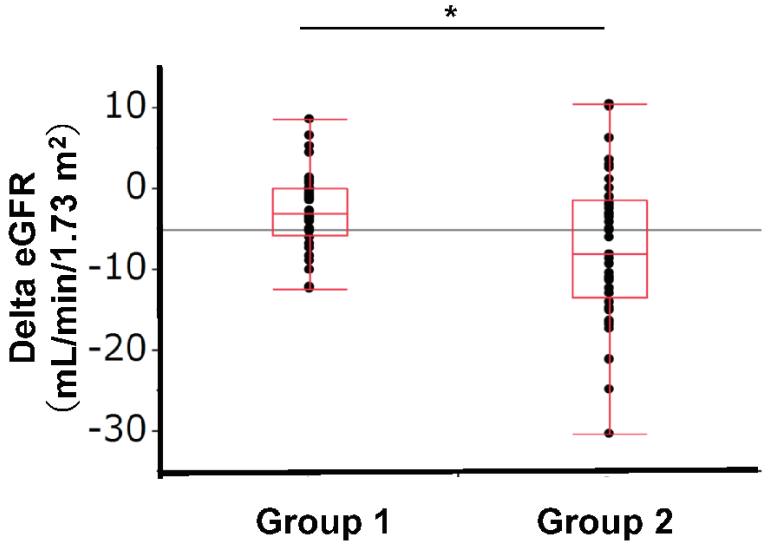

b

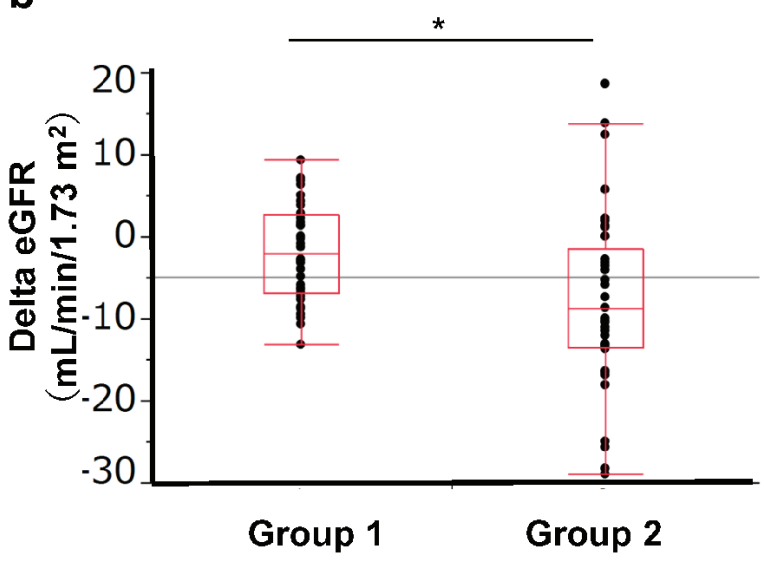

Figure 1. Change in eGFR according to baseline eGFR group. Group 1: eGFR $<80.0 \mathrm{~mL} / \mathrm{min} / 1.73 \mathrm{~m}{ }^{2}$, Group 2: eGFR $\geq 80 \mathrm{~mL} /$ $\mathrm{min} / 1.73 \mathrm{~m}^{2}$. (a) Change in eGFR from baseline to 4 weeks. (b) Change in eGFR from baseline to 12 weeks.

analyses showed that the changes in eGFR were not affected by age, sex, body weight change, or the use of ACE inhibitor or ARB and were correlated with the eGFR at baseline (Table 2 ). We divided the patients into two groups according to the median baseline eGFR $\left(80.0 \mathrm{~mL} / \mathrm{min} / 1.73 \mathrm{~m}^{2}\right)$ : Group 1 was defined as those with a baseline eGFR below $80 \mathrm{~mL} / \mathrm{min} / 1.73$ $\mathrm{m}^{2}$, while subjects with a baseline eGFR of more than $80 \mathrm{~mL} /$ $\min / 1.73 \mathrm{~m}^{2}$ were assigned to Group 2. No significant differences in the baseline characteristics were observed between the two groups except for renal functions (BUN, Cr, eGFR, and CCr) (Supplementary Table 1) (www.jofem.org). Group 2 had a greater reduction in eGFR at 4 weeks than that in Group 1 (Fig. 1). Although Group 2 showed a significant improvement in HbA1c compared with Group 1 at 4 weeks, no significant difference was observed at 12 weeks (Table 3). The lipid parameters (LDL-cho, HDL-cho, and TG) and uric acid value were not significantly different between the two groups at either 4 or 12 weeks.

Effects of SGLT2 inhibition on eGFR and glomerular and tubular damage markers in patients with type 2 diabetes in a longitudinal study

Next, we investigated the changes in glomerular damage markers after treatment with SGLT-2 inhibitors in type 2 diabetes patients. The urinary NAG, L-FABP, type IV collagen, and $\beta 2 \mathrm{MG}$ levels were measured in 23 of the 81 above-mentioned patients. Supplementary Table 2 (www.jofem.org) shows the results after 4 weeks of SGLT-2 inhibitor treatment in 23 type 2 diabetes patients (14 men and nine women; mean age, $51.7 \pm 11.9$ years old; mean duration of diabetes, $9.78 \pm 7.34$ years) with nor-

Table 3. Mean Changes From Baseline in Glycemic Control, Lipid Profile, and Renal Function in Patients Receiving SGLT2 Inhibitors for 12 Weeks by Baseline eGFR Group $(\mathrm{N}=81)$

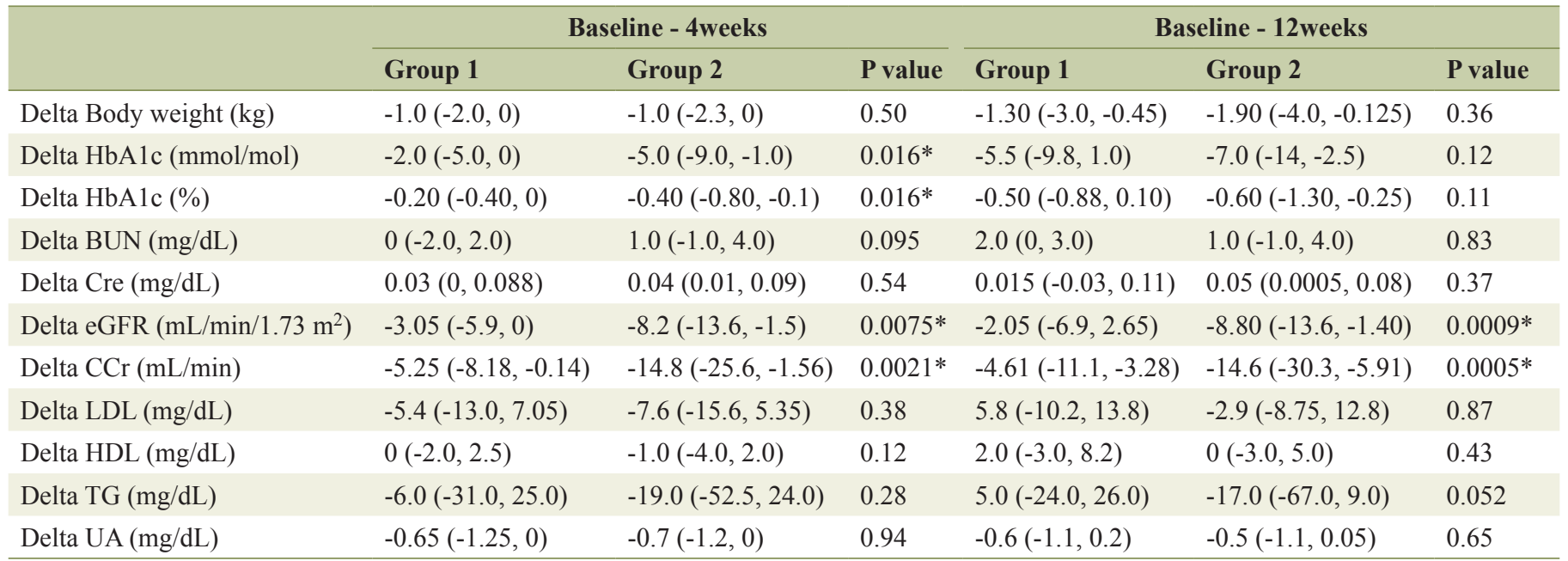

Medians (25th and 75th percentiles). CCr was calculated by Cockcroft and Gault equation. P values were determined by Mann-Whitney U test. *P $<0.05$. 
Table 4. Mean Changes From Baseline in Glycemic Control, Blood Pressure, Lipid Profile, and Renal Function in Patients Receiving SGLT-2 Inhibitors for 4 Weeks and 12 Weeks $(\mathrm{N}=19)$

\begin{tabular}{|c|c|c|c|c|c|c|c|}
\hline & & & & & & P value & \\
\hline & $\begin{array}{l}\text { Standard } \\
\text { value }\end{array}$ & Baseline & 4 weeks & 12 weeks & $\begin{array}{l}\text { O vs. } 4 \\
\text { weeks }\end{array}$ & $\begin{array}{l}4 \text { vs. } 12 \\
\text { weeks }\end{array}$ & $\begin{array}{l}0 \text { vs. } 12 \\
\text { weeks }\end{array}$ \\
\hline Body mass index $\left(\mathrm{kg} / \mathrm{m}^{2}\right)$ & & $28.8 \pm 3.51$ & $28.6 \pm 3.38$ & $27.9 \pm 3.67$ & 0.44 & $0.0094 *$ & $0.0043 *$ \\
\hline $\mathrm{HbAlc}(\mathrm{mmol} / \mathrm{mol})$ & & $68.6 \pm 16.4$ & $60.8 \pm 9.28$ & $54.6 \pm 8.86$ & $0.0022 *$ & $0.012 *$ & $0.0031 *$ \\
\hline $\mathrm{CCr}(\mathrm{mL} / \mathrm{min})$ & & $127 \pm 26.7$ & $122 \pm 29.5$ & $120 \pm 29.7$ & 0.12 & 0.37 & $0.048 *$ \\
\hline $\mathrm{SBP}(\mathrm{mm} \mathrm{Hg})$ & & $135 \pm 19.6$ & $130 \pm 16.0$ & $128 \pm 15.7$ & 0.073 & 0.43 & $0.024 *$ \\
\hline $\mathrm{DBP}(\mathrm{mm} \mathrm{Hg})$ & & $81.6 \pm 14.3$ & $80.7 \pm 11.6$ & $78.7 \pm 10.9$ & 0.51 & 0.61 & 0.27 \\
\hline LDL-C (mg/dL) & & $81.0(72.2,110)$ & $74.8(64.0,83.2)$ & $76.8(64.2,91.4)$ & 0.074 & 0.28 & 0.52 \\
\hline Urinary albumin (mg/g Cre) & $\leq 18.6$ & $12.0(6.6,56.2)$ & $12.1(7.5,28.7)$ & $13.3(6.3,30.5)$ & 0.52 & 0.059 & 0.078 \\
\hline Urinary NAG (U/g Cre) & $\leq 5.6$ & $5.97(2.67,7.79)$ & $6.33(5.31,9.34)$ & $5.83(4.73,7.88)$ & 0.11 & 0.096 & 0.52 \\
\hline Urinary L-FABP $(\mu \mathrm{g} / \mathrm{g}$ Cre $)$ & $\leq 8.4$ & $2.70(1.30,5.80)$ & $2.91(1.90,4.00)$ & $2.30(1.30,4.16)$ & 0.4 & 0.65 & 0.77 \\
\hline Urinary type IV collagen ( $\mu \mathrm{g} / \mathrm{g}$ Cre) & $\leq 7.3$ & $5.40(2.00,7.20)$ & $5.30(3.80,6.70)$ & $4.60(3.70,6.50)$ & 0.19 & 0.52 & 0.71 \\
\hline Urinary $\beta 2 \mathrm{MG}(\mu \mathrm{g} / \mathrm{g}$ Cre $)$ & $\dagger$ & $78.6(40.0,181)$ & $111(65.9,173)$ & $133(100,243)$ & 0.95 & 0.12 & 0.12 \\
\hline
\end{tabular}

Mean \pm standard deviation or medians (25th and 75th percentiles). CCr was calculated by Cockcroft and Gault equation. $†$ The reference value of $\beta 2$ microglobulin was $11-253 \mu \mathrm{g} /$ day in our hospital. We used the spot urine sample and the urinary excretion levels of $\beta 2$ microglobulin were described as micrograms per gram of creatinine. $P$ values were determined by paired $t$-test or Wilcoxon signed-rank test. NAG: N-acetyl- $\beta$-D-glucosaminidase; L-FABP: liver-type fatty acid binding protein; $\beta 2 \mathrm{MG}$ : $\beta 2$ microglobulin. ${ }^{*} \mathrm{P}<0.05$.

moalbuminuria $(n=16)$, microalbuminuria $(n=3)$, or macroalbuminuria $(\mathrm{n}=4)$. The HbA1c, eGFR, CCr, and UA levels were significantly reduced after treatment with the SGLT2 inhibitor at week 4 (Supplementary Table 2) (www.jofem.org). Of note, the urinary NAG $(\mathrm{P}=0.053)$, L-FABP $(\mathrm{P}=0.10)$, type IV collagen $(\mathrm{P}=0.024)$, and $\beta 2 \mathrm{MG}(\mathrm{P}=0.57)$ levels showed a tendency to be elevated. Nineteen (12 men and seven women; mean age, $52.6 \pm 11.0$ years old; mean duration of diabetes, $10.8 \pm 7.42$ years) of the 23 above-mentioned patients were also followed up after 12 weeks of treatment (Table 4). The previously observed trends in the eGFR and glomerular and tubular damage markers at 4 weeks of treatment had almost recovered to the baseline levels after 12 weeks of treatment, although the body weight, HbA1c, SBP and UA levels were still significantly reduced by the SGLT2 inhibitor at week 12 (Table 4).

\section{Discussion}

In this study, we investigated the effects of SGLT-2 inhibition on the eGFR and other glomerular damage markers in Japanese type 2 diabetes patients. Our data showed that the initial reduction in eGFR induced by treatment with SGLT2 inhibitors was greater in the subjects who had a higher baseline eGFR. Similar to previous reports, the initial reduction in
eGFR tended to recover to the baseline level at 12 weeks after SGLT2 inhibitor treatment. NAG, L-FABP, type IV collagen, and $\beta 2 \mathrm{MG}$ also showed a tendency to increase transiently after the start of SGLT-2 inhibition in type 2 diabetes patients.

In type 2 diabetes patients with hypertension receiving renin-angiotensin blockers, the initial reduction in eGFR in subjects receiving dapagliflozin was completely reversible after the discontinuation of treatment [11]. Therefore, the initial fall in eGFR as a result of SGLT-2 inhibitor treatment was not thought to reflect a reduction in the number of functioning nephron GFR. In this study, a higher eGFR at baseline was correlated with a greater reduction in eGFR at both 4 and 12 weeks after the start of SGLT2 inhibitor treatment, compared with the lower eGFR group, independent of the changes in $\mathrm{HbA1c}$ and uric acid. This result possibly suggested that the baseline eGFR affects the renoprotective action of SGLT2 inhibitors in the context of correcting hyperfiltration.

Experimental studies have suggested that SGLT2 inhibitors ameliorate glomerular hyperfiltration and reduce albuminuria, kidney hypertrophy and inflammation in animal models of type 1 and type 2 diabetes $[3,15]$. The results of the EMPAREG OUTCOME trial suggested that SGLT2 inhibition provides renoprotection by decreasing hyperfiltration [6], arterial stiffness [16, 17], vascular resistance [17], serum uric acid levels [18], and systemic and renal neurohormonal systems [16, 
19] in type 2 diabetes patients, despite a reduction in the eGFR. The initial reduction in eGFR induced by SGLT2 inhibitors is thought to be associated with a reduction in blood pressure, an osmotic diuretic effect, and TGF $[1,20]$. The present study also showed an initially significant decrease in eGFR, blood pressure, and uric acid levels after 4 weeks of treatment. While empagliflozin has a protective effect on inflammatory and fibrotic marker expressions in human proximal tubular cells [4], our results showing a transient increase in glomerular and tubular damage markers because of SGLT2 inhibition is a new observation for this emerging diabetes treatment. Even if the transient increase in glomerular and tubular damage markers reflects glomerular and tubulointerstitial injuries, the changes observed in this study were almost within the normal range. Therefore, the clinical significance of these transient changes might be minimal, and the changes may reflect a transitory change in eGFR. Further consideration should be given to the transient changes in renal function markers during treatment with SGLT2 inhibitors.

The limitations of this study include a small sample size and a relatively short follow-up period. Furthermore, a control group was not examined, and the biomarker data did not exhibit normal distributions and had large degrees of variance.

In summary, the results of our investigation suggested that SGLT2 inhibition reduces the eGFR over the short term, is correlated with the baseline eGFR level, and tends to have recovered to the baseline level after 12 weeks of treatment in type 2 diabetes patients. SGLT2 inhibition may increase glomerular and tubular damage markers initially, but these markers eventually recover to their baseline levels. Further studies are needed to evaluate the effects of SGLT2 inhibitors on renal functions.

\section{Acknowledgments}

We thank Drs. Saho Hosokawa, Hirotatsu Nakaguchi and Kazuki Orime (Yokohama City University) for their assistance, and Ms. Misa Katayama (Yokohama City University) for her secretarial assistance.

\section{Grant Support}

This work was supported in part by Grants-in-Aid for Scientific Research (B) 16H05329 from the Ministry of Education, Culture, Sports, Science and Technology of Japan (to Y.Te.).

\section{Conflict of Interest}

None of the authors has any potential conflict of interest associated with this research.

\section{Abbreviations}

SGLT2 inhibitors: sodium-glucose cotransporter 2 inhibi- tors; eGFR: estimated glomerular filtration rate; TGF: tubuloglomerular feedback; NAG: N-acetyl- $\beta$-D-glucosaminidase; L-FABP: liver-type fatty acid binding protein; $\beta 2 \mathrm{MG}$ : $\beta 2 \mathrm{mi}-$ croglobulin; LDL-cholesterol: low-density lipoprotein-cholesterol; HDL-cholesterol: high-density lipoprotein-cholesterol

\section{References}

1. Wanner C, Inzucchi SE, Lachin JM, Fitchett D, von Eynatten M, Mattheus M, Johansen OE, et al. Empagliflozin and progression of kidney disease in type 2 diabetes. N Engl J Med. 2016;375(4):323-334.

2. Cherney DZ, Scholey JW, Jiang S, Har R, Lai V, Sochett $\mathrm{EB}$, Reich HN. The effect of direct renin inhibition alone and in combination with ACE inhibition on endothelial function, arterial stiffness, and renal function in type 1 diabetes. Diabetes Care. 2012;35(11):2324-2330.

3. De Nicola L, Gabbai FB, Liberti ME, Sagliocca A, Conte G, Minutolo R. Sodium/glucose cotransporter 2 inhibitors and prevention of diabetic nephropathy: targeting the renal tubule in diabetes. Am J Kidney Dis. 2014;64(1):1624.

4. Panchapakesan U, Pegg K, Gross S, Komala MG, Mudaliar H, Forbes J, Pollock C, et al. Effects of SGLT2 inhibition in human kidney proximal tubular cellsrenoprotection in diabetic nephropathy? PLoS One. 2013;8(2):e54442.

5. Cherney DZ, Perkins BA, Soleymanlou N, Maione M, Lai $\mathrm{V}$, Lee A, Fagan NM, et al. Renal hemodynamic effect of sodium-glucose cotransporter 2 inhibition in patients with type 1 diabetes mellitus. Circulation. 2014;129(5):587597.

6. Vallon V, Gerasimova M, Rose MA, Masuda T, Satriano J, Mayoux E, Koepsell H, et al. SGLT2 inhibitor empagliflozin reduces renal growth and albuminuria in proportion to hyperglycemia and prevents glomerular hyperfiltration in diabetic Akita mice. Am J Physiol Renal Physiol. 2014;306(2):F194-204.

7. Magee GM, Bilous RW, Cardwell CR, Hunter SJ, Kee F, Fogarty DG. Is hyperfiltration associated with the future risk of developing diabetic nephropathy? A meta-analysis. Diabetologia. 2009;52(4):691-697.

8. Huang HC, Preisig PA. G1 kinases and transforming growth factor-beta signaling are associated with a growth pattern switch in diabetes-induced renal growth. Kidney Int. 2000;58(1):162-172.

9. Bak M, Thomsen K, Christiansen T, Flyvbjerg A. Renal enlargement precedes renal hyperfiltration in early experimental diabetes in rats. J Am Soc Nephrol. 2000;11(7):1287-1292.

10. Thomson SC, Rieg T, Miracle C, Mansoury H, Whaley J, Vallon V, Singh P. Acute and chronic effects of SGLT2 blockade on glomerular and tubular function in the early diabetic rat. Am J Physiol Regul Integr Comp Physiol. 2012;302(1):R75-83.

11. Heerspink HJ, Johnsson E, Gause-Nilsson I, Cain VA, Sjostrom CD. Dapagliflozin reduces albuminuria in patients with diabetes and hypertension receiv- 
ing renin-angiotensin blockers. Diabetes Obes Metab. 2016;18(6):590-597.

12. Yale JF, Bakris G, Cariou B, Yue D, David-Neto E, Xi L, Figueroa K, et al. Efficacy and safety of canagliflozin in subjects with type 2 diabetes and chronic kidney disease. Diabetes Obes Metab. 2013;15(5):463-473.

13. Kaku K, Lee J, Mattheus M, Kaspers S, George J, Woerle HJ, Investigators E-RO. Empagliflozin and cardiovascular outcomes in asian patients with type 2 diabetes and established cardiovascular disease- results from EMPAREG OUTCOME((R)). Circ J. 2017;81(2):227-234.

14. Matsuo S, Imai E, Horio M, Yasuda Y, Tomita K, Nitta $\mathrm{K}$, Yamagata $\mathrm{K}$, et al. Revised equations for estimated GFR from serum creatinine in Japan. Am J Kidney Dis. 2009;53(6):982-992.

15. Gembardt F, Bartaun C, Jarzebska N, Mayoux E, Todorov VT, Hohenstein B, Hugo C. The SGLT2 inhibitor empagliflozin ameliorates early features of diabetic nephropathy in BTBR ob/ob type 2 diabetic mice with and without hypertension. Am J Physiol Renal Physiol. 2014;307(3):F317-325.

16. Cherney DZ, Perkins BA, Soleymanlou N, Har R, Fagan $\mathrm{N}$, Johansen OE, Woerle HJ, et al. The effect of empa- gliflozin on arterial stiffness and heart rate variability in subjects with uncomplicated type 1 diabetes mellitus. Cardiovasc Diabetol. 2014;13:28.

17. Chilton R, Tikkanen I, Cannon CP, Crowe S, Woerle HJ, Broedl UC, Johansen OE. Effects of empagliflozin on blood pressure and markers of arterial stiffness and vascular resistance in patients with type 2 diabetes. Diabetes Obes Metab. 2015;17(12):1180-1193.

18. Zinman B, Wanner C, Lachin JM, Fitchett D, Bluhmki E, Hantel S, Mattheus M, et al. Empagliflozin, Cardiovascular Outcomes, and Mortality in Type 2 Diabetes. N Engl J Med. 2015;373(22):2117-2128.

19. Cherney DZ, Perkins BA, Soleymanlou N, Xiao F, Zimpelmann J, Woerle HJ, Johansen OE, et al. Sodium glucose cotransport-2 inhibition and intrarenal RAS activity in people with type 1 diabetes. Kidney Int. 2014;86(5):1057-1058

20. Leiter LA, Yoon KH, Arias P, Langslet G, Xie J, Balis DA, Millington D, et al. Canagliflozin provides durable glycemic improvements and body weight reduction over 104 weeks versus glimepiride in patients with type 2 diabetes on metformin: a randomized, double-blind, phase 3 study. Diabetes Care. 2015;38(3):355-364. 